\title{
Note on the Degenerate Gamma Function
}

\author{
T. Kim* ${ }^{*, 1}$ and D. S. Kim**,2 \\ *Department of Mathematics, Kwangwoon University, Seoul 139-701, Republic of Korea, \\ ** Department of Mathematics, Sogang University, Seoul 121-742, Republic of Korea, \\ E-mail: ${ }^{1}$ tkkim@kw.ac.kr, ${ }^{2}$ dskim@sogang.ac.kr
}

Received April 10, 2020; Revised April 15, 2020; Accepted April 17, 2020

\begin{abstract}
Recently, the degenerate gamma functions were introduced as a degenerate version of the usual gamma function. In this paper, we investigate several properties of these functions. Namely, we obtain an analytic continuation as a meromorphic function on the whole complex plane, the difference formula, the values at positive integers, some expressions following from the Weierstrass and Euler formulas for the ordinary gamma function, and an integral representation as an integral along a Hankel contour.
\end{abstract}

DOI 10.1134/S1061920820030061

\section{INTRODUCTION}

It is not an exaggeration to say that the gamma function is the most important nonelementary transcendental function. It appears in many areas, such as hypergeometric series, asymptotic series, definite integration, Riemann zeta function, $L$-functions, and number theory in general. The gamma function was introduced by Euler and subsequently studied by eminent mathematicians like Daniel Bernoulli, Legendre, Gauss, Liouville, Weierstrass, Hermite, as well as many other mathematicians.

In [6], the degenerate gamma functions were introduced, as an attempt to find a degenerate version of the ordinary gamma function, and the related degenerate Laplace transform was also studied (see also [7]). In this paper, we would like to derive some basic properties of the degenerate gamma functions including the analytic continuation to a meromorphic function on the whole complex plane, the difference formula, some expressions coming from the Euler and Weierstrass formulas, and an integral representation as an integral along a Hankel contour. We also discuss degenerate beta functions.

In the rest of this section, we recall some basic facts on the gamma function, the beta function, the degenerate exponential function, and the degenerate gamma functions. In Sec. 2, we prove the main results of this paper. Finally, we discuss an integral representation of the degenerate gamma functions in Sec. 3 and conclude our results in Sec. 4. Recently, L. M. Upadhyaya published many papers on the degenerate Laplace transforms(see [20]: International Journal of Engineering, Science and Mathematics). However, almost all of his studies are directly derived from the results previously studied by Kim-Kim in [6]. A good example of the application of a gamma function in this paper, is the construction of degenerate gamma random variables used to predict corona viruses (see [11]). It is very difficult to predict the number of people transmitting the virus within a given period of time at a time when the coronavirus is rapidly transmitted in accordance to sudden variables (see [11]). The degenerate gamma function was used to construct a well-used degenerate gamma random variable in these circumstances.

For $s \in \mathbb{C}$ with $\operatorname{Re}(s)>0$, the gamma function is defined by

$$
\left.\Gamma(s)=\int_{0}^{\infty} e^{-t} t^{s-1} d t \quad \text { see } \quad[1-22]\right) .
$$

It follows from (1.1) that

$$
\Gamma(s+1)=s \Gamma(s), \quad \Gamma(n)=(n-1) ! \quad(n \in \mathbb{N}) \quad(\text { see }[15,16,17,18,19]) .
$$

For $\alpha, \beta \in \mathbb{C}$ with $\operatorname{Re}(\alpha)>0$ and $\operatorname{Re}(\beta)>0$, the beta function is defined by the integrals

$$
B(\alpha, \beta)=\int_{0}^{1} t^{\alpha-1}(1-t)^{\beta-1} d t=\int_{0}^{\infty} \frac{u^{\alpha-1}}{(1+u)^{\alpha+\beta}} d u \quad(\text { see }[1,15,16,17]) .
$$

It follows from (1.1) and (1.3) that

$$
B(\alpha, \beta)=\frac{\Gamma(\alpha) \Gamma(\beta)}{\Gamma(\alpha+\beta)} \quad(\text { see }[15,22]) .
$$


As is well known, the Euler's formula for the gamma function is given by

$$
\begin{aligned}
\Gamma(z) & =\frac{1}{z} \prod_{n=1}^{\infty}\left\{\left(1+\frac{1}{n}\right)^{z}\left(1+\frac{z}{n}\right)^{-1}\right\} \\
& \left.=\lim _{n \rightarrow \infty} \frac{(n-1) !}{z(z+1) \cdots(z+n-1)} n^{z} \quad \text { (see }[2,3,4,13,16,22]\right),
\end{aligned}
$$

where $z \neq 0,-1,-2,-3, \cdots$. Using (1.5), we can derive the Weierstrass formula which is given by

$$
\frac{1}{\Gamma(z)}=z e^{\gamma z} \prod_{n=1}^{\infty}\left\{\left(1+\frac{z}{n}\right) e^{-\frac{z}{n}}\right\} \quad(\text { see }[2,3,4,22]) .
$$

where $\gamma$ is the Euler constant defined by

$$
\gamma=\lim _{n \rightarrow \infty}\left(1+\frac{1}{2}+\frac{1}{3} \cdots+\frac{1}{n}-\log n\right) .
$$

Note here that we may speak of the analytically continued beta function obtained from (1.4), which is analytic except for $\alpha=0,-1,-2, \ldots, \beta=0,-1,-2, \ldots$ Indeed, from (1.4) and (1.6) we have

$$
B(\alpha, \beta)=\frac{\Gamma(\alpha) \Gamma(\beta)}{\Gamma(\alpha+\beta)}=\frac{\alpha+\beta}{\alpha \beta} \prod_{n=1}^{\infty} \frac{\left(1+\frac{\alpha+\beta}{n}\right)}{\left(1+\frac{\alpha}{n}\right)\left(1+\frac{\beta}{n}\right)} .
$$

It follows from (1.6) that

$$
\begin{aligned}
\Gamma(z) \Gamma(-z) & =-\frac{1}{z^{2}} \prod_{n=1}^{\infty}\left\{\left(1+\frac{z}{n}\right) e^{-\frac{z}{n}}\right\}^{-1} \prod_{n=1}^{\infty}\left\{\left(1-\frac{z}{n}\right) e^{\frac{z}{n}}\right\}^{-1} \\
& =\frac{-\pi}{z \sin \pi z}
\end{aligned}
$$

Thus, by (1.7) and (1.2), we obtain

$$
\Gamma(z) \Gamma(1-z)=\Gamma(z)(-z \Gamma(-z))=\frac{\pi}{\sin \pi z} \quad(\text { see }[16])
$$

For any nonzero $\lambda \in \mathbb{R}$, the degenerate exponential functions are defined as

$$
e_{\lambda}^{x}(t)=(1+\lambda t)^{\frac{x}{\lambda}}, \quad e_{\lambda}(t)=e_{\lambda}^{1}(t)=(1+\lambda t)^{\frac{1}{\lambda}} \quad(\text { see }[6,7,8,9,10]) .
$$

Note that $\lim _{\lambda \rightarrow 0} e_{\lambda}^{x}(t)=e^{x t}$.

Let $\log _{\lambda}(t)$ be the compositional inverse of $e_{\lambda}(t)$, which is called the degenerate logarithm. Then we have

$$
\log _{\lambda}(t)=\frac{1}{\lambda}\left(t^{\lambda}-1\right) \quad(\text { see }[8,9,10]) .
$$

It follows from (1.9) and (1.10) that

$$
t=\log _{\lambda} e_{\lambda}(t)=e_{\lambda} \log _{\lambda}(t)
$$

For $\lambda \in(0,1)$, the degenerate gamma function for a complex variable $s$ with $0<\operatorname{Re}(s)<\frac{1}{\lambda}$ is defined by Kim-Kim as

$$
\Gamma_{\lambda}(s)=\int_{0}^{\infty} e_{\lambda}^{-1}(t) t^{s-1} d t=\int_{0}^{\infty}(1+\lambda t)^{-\frac{1}{\lambda}} t^{s-1} d t \quad(\text { see }[6]) .
$$

Thus, by (1.11), we obtain

$$
\Gamma_{\lambda}(s+1)=\frac{s}{(1-\lambda)^{s+1}} \Gamma_{\frac{\lambda}{1-\lambda}}(s), \quad 0<\operatorname{Re}(s)<\frac{1-\lambda}{\lambda} \quad(\text { see }[6,7,11,12,20]) .
$$


It follows from (1.12) that, for any integer $k \geqslant 0$, we have

$$
\begin{aligned}
\Gamma_{\lambda}(s+1)= & \frac{s(s-1)(s-2) \cdots(s-(k+1)+1)}{(1-\lambda)(1-2 \lambda) \cdots(1-k \lambda)(1-(k+1) \lambda)^{s-k+1}} \\
& \times \Gamma_{\frac{\lambda}{1-(k+1) \lambda}}(s-k) \quad(\text { see }[6]),
\end{aligned}
$$

where $\lambda \in\left(0, \frac{1}{k+1}\right)$ with $k \in \mathbb{N}$ and $k<\operatorname{Re}(s)<\frac{1-\lambda}{\lambda}$. In particular, for $k \in \mathbb{N}$ and $\lambda \in\left(0, \frac{1}{k}\right)$, we have

$$
\Gamma_{\lambda}(k)=\frac{(k-1) !}{(1-\lambda)(1-2 \lambda) \cdots(1-k \lambda)} \quad(\text { see }[6]) .
$$

\section{DEGENERATE GAMMA FUNCTIONS}

In this section, we assume that $\lambda$ is any chosen real number with $0<\lambda<1$. By (1.3), we have

$$
\Gamma_{\lambda}(s)=\lambda^{-s} \int_{0}^{\infty} \frac{t^{s-1}}{(1+t)^{\frac{1}{\lambda}}} d t=\lambda^{-s} B\left(s, \frac{1}{\lambda}-s\right)=\lambda^{-s} \frac{\Gamma(s) \Gamma\left(\frac{1}{\lambda}-s\right)}{\Gamma\left(\frac{1}{\lambda}\right)} .
$$

Note here that (2.1) holds initially for $s \in \mathbb{C}$ with $0<\operatorname{Re}(s)<\frac{1}{\lambda}$ and that it further holds for any $s \in \mathbb{C} \backslash\left\{0,-1,-2, \ldots, \frac{1}{\lambda}, \frac{1}{\lambda}+1, \frac{1}{\lambda}+2, \ldots\right\}$ by the analytic continuation, and defines an analytic function on $\mathbb{C} \backslash\left\{0,-1,-2, \ldots, \frac{1}{\lambda}, \frac{1}{\lambda}+1, \frac{1}{\lambda}+2, \ldots\right\}$. Moreover, it has simple poles at $s=-n,(n=0,1,2 \ldots)$ with the residues

$$
\operatorname{res}_{s=-n}=\frac{(-1)^{n} \lambda^{n} \Gamma\left(\frac{1}{\lambda}+n\right)}{n ! \Gamma\left(\frac{1}{\lambda}\right)},
$$

and simple poles at $s=\frac{1}{\lambda}+n,(n=0,1,2 \ldots)$ with the residues

$$
\operatorname{res}_{s=\frac{1}{\lambda}+n}=\frac{(-1)^{n-1} \lambda^{-n-\frac{1}{\lambda}} \Gamma\left(\frac{1}{\lambda}+n\right)}{n ! \Gamma\left(\frac{1}{\lambda}\right)} .
$$

Therefore, by (2.1), we obtain the following lemma.

Lemma 2.1. Let $\lambda \in(0,1)$. Then $\Gamma_{\lambda}(s)$, which is initially defined for $0<\operatorname{Re}(s)<\frac{1}{\lambda}$, has an analytic continuation to a meromorphic function on $\mathbb{C}$ whose only singularities are simple poles at $s=0,-1,-2, \ldots, \frac{1}{\lambda}, \frac{1}{\lambda}+1, \frac{1}{\lambda}+2, \ldots$, with the residues

$$
\operatorname{res}_{s=-n}=\frac{(-1)^{n} \lambda^{n} \Gamma\left(\frac{1}{\lambda}+n\right)}{n ! \Gamma\left(\frac{1}{\lambda}\right)}, \quad \operatorname{res}_{s=\frac{1}{\lambda}+n}=\frac{(-1)^{n-1} \lambda^{-n-\frac{1}{\lambda}} \Gamma\left(\frac{1}{\lambda}+n\right)}{n ! \Gamma\left(\frac{1}{\lambda}\right)} .
$$

In addition, the following identities hold for any $s \in \mathbb{C} \backslash\left\{0,-1,-2, \ldots, \frac{1}{\lambda}, \frac{1}{\lambda}+1, \frac{1}{\lambda}+2, \ldots\right\}$ :

$$
\begin{gathered}
\Gamma_{\lambda}(s)=\lambda^{-s} \frac{\Gamma(s) \Gamma\left(\frac{1}{\lambda}-s\right)}{\Gamma\left(\frac{1}{\lambda}\right)}=\lambda^{-s} B\left(s, \frac{1}{\lambda}-s\right), \\
\lambda^{s} \Gamma_{\lambda}(s)=\lambda^{\frac{1}{\lambda}-s} \Gamma_{\lambda}\left(\frac{1}{\lambda}-s\right) .
\end{gathered}
$$

It follows from Lemma 2.1 that

$$
\begin{aligned}
\Gamma_{\lambda}(s+1) & =\lambda^{-s-1} \frac{\Gamma(s+1) \Gamma\left(\frac{1}{\lambda}-s-1\right)}{\Gamma\left(\frac{1}{\lambda}\right)}=\frac{s \lambda^{-s}}{\lambda\left(\frac{1}{\lambda}-s-1\right)} \frac{\Gamma(s)\left(\frac{1}{\lambda}-s-1\right) \Gamma\left(\frac{1}{\lambda}-s\right)}{\Gamma\left(\frac{1}{\lambda}\right)} \\
& =\frac{s}{\lambda\left(\frac{1}{\lambda}-s-1\right)} \lambda^{-s} \frac{\Gamma(s) \Gamma\left(\frac{1}{\lambda}-s\right)}{\Gamma\left(\frac{1}{\lambda}\right)}=\frac{s}{1-\lambda(s+1)} \Gamma_{\lambda}(s) .
\end{aligned}
$$

By (1.11) and (2.4), we obtain the following theorem. 
Theorem 2.2. Let $\lambda \in(0,1)$. Then the following difference equation holds for any

$$
\begin{gathered}
s \in \mathbb{C} \backslash\left\{0,-1,-2 \ldots, \frac{1}{\lambda}-1, \frac{1}{\lambda}, \frac{1}{\lambda}+1, \ldots\right\}: \\
\Gamma_{\lambda}(s+1)=\frac{s}{1-\lambda(s+1)} \Gamma_{\lambda}(s) .
\end{gathered}
$$

Let $s=k$ be a positive integer. Then, by Theorem 2.2 , we obtain

$$
\begin{aligned}
\Gamma_{\lambda}(k+1) & =\frac{k}{1-\lambda(k+1)} \Gamma_{\lambda}(k)=\frac{k(k-1)}{(1-\lambda(k+1))(1-\lambda k)} \Gamma_{\lambda}(k-1) \\
& =\cdots=\frac{k \cdot(k-1) \cdots 2 \cdot 1}{(1-(k+1) \lambda)(1-k \lambda) \cdots(1-2 \lambda)} \Gamma_{\lambda}(1) .
\end{aligned}
$$

By (2.1), we have

$$
\Gamma_{\lambda}(1)=\frac{1}{\lambda} \frac{\Gamma(1) \Gamma\left(\frac{1}{\lambda}-1\right)}{\Gamma\left(\frac{1}{\lambda}\right)}=\frac{1}{\lambda\left(\frac{1}{\lambda}-1\right)}=\frac{1}{1-\lambda}
$$

Thus, by (2.5) and (2.6), for any integer $k \geqslant 0$ we obtain

$$
\Gamma_{\lambda}(k+1)=\frac{\Gamma(k+1)}{(1)_{k+2, \lambda}},
$$

where $(x)_{0, \lambda}=1,(x)_{n, \lambda}=x(x-\lambda) \cdots(x-(n-1) \lambda),(n \geqslant 1),($ see $[5-11])$.

Therefore, by (2.7), we obtain the following theorem.

Theorem 2.3. Let $\lambda \in(0,1)$. Then, for $k \in \mathbb{N}$, we have

$$
\Gamma_{\lambda}(k)=\frac{\Gamma(k)}{(1)_{k+1, \lambda}} .
$$

It follows from (1.6) and Lemma 2.1 that

$$
\begin{aligned}
\frac{1}{\Gamma_{\lambda}(z)}= & \Gamma\left(\frac{1}{\lambda}\right) \lambda^{z} \frac{1}{\Gamma(z)} \frac{1}{\Gamma\left(\frac{1}{\lambda}-z\right)}=\lambda^{z} \Gamma\left(\frac{1}{\lambda}\right) z e^{\gamma z} \prod_{n=1}^{\infty}\left\{\left(1+\frac{z}{n}\right) e^{-\frac{z}{n}}\right\}\left(\frac{1}{\lambda}-z\right) e^{\gamma\left(\frac{1}{\lambda}-z\right)} \\
& \times \prod_{n=1}^{\infty}\left\{\left(1+\frac{\frac{1}{\lambda}-z}{n}\right) e^{-\frac{1}{\lambda}-z}\right\}=\lambda^{z} \Gamma\left(\frac{1}{\lambda}\right) z\left(\frac{1}{\lambda}-z\right) e^{\frac{\gamma}{\lambda}} \prod_{n=1}^{\infty}\left\{e^{-\frac{1}{n \lambda}}\left(1+\frac{z}{n}\right)\left(1+\frac{\frac{1}{\lambda}-z}{n}\right)\right\} .
\end{aligned}
$$

It is also clear from this equation that $\Gamma_{\lambda}(z)$ is analytic on $\mathbb{C}$ except for the points

$$
z=0,-1,-2, \ldots, \frac{1}{\lambda}, \frac{1}{\lambda}+1, \frac{1}{\lambda}+2, \ldots
$$

at which it has simple poles.

Thus, by (2.8), we obtain

$$
\begin{aligned}
\frac{1}{\Gamma_{\lambda}(z)} & =\lambda^{z} \Gamma\left(\frac{1}{\lambda}\right) z\left(\frac{1}{\lambda}-z\right) \lim _{m \rightarrow \infty} e^{\frac{1}{\lambda}\left(1+\frac{1}{2}+\cdots+\frac{1}{m}-\log m\right)}\left[\lim _{m \rightarrow \infty} \prod_{n=1}^{m} e^{-\frac{1}{n \lambda}}\left(1+\frac{z}{n}\right)\left(1+\frac{\frac{1}{\lambda}-z}{n}\right)\right] \\
& =\lambda^{z} z\left(\frac{1}{\lambda}-z\right) \Gamma\left(\frac{1}{\lambda}\right) \lim _{m \rightarrow \infty}\left[\prod_{n=1}^{m} m^{-\frac{1}{\lambda}}\left(1+\frac{z}{n}\right)\left(1+\frac{\frac{1}{\lambda}-z}{n}\right)\right] \\
& =\lambda^{z} z\left(\frac{1}{\lambda}-z\right) \Gamma\left(\frac{1}{\lambda}\right) \lim _{m \rightarrow \infty}\left[\prod_{n=1}^{m-1}\left(1+\frac{1}{n}\right)^{-\frac{1}{\lambda}}\right]\left[\prod_{n=1}^{m}\left(1+\frac{z}{n}\right)\left(1+\frac{\frac{1}{\lambda}-z}{n}\right)\right] \\
& =\lambda^{z} z\left(\frac{1}{\lambda}-z\right) \Gamma\left(\frac{1}{\lambda}\right) \prod_{n=1}^{\infty}\left\{\left(1+\frac{1}{n}\right)^{-\frac{1}{\lambda}}\left(1+\frac{z}{n}\right)\left(1+\frac{\frac{1}{\lambda}-z}{n}\right)\right\}
\end{aligned}
$$

Therefore, by (2.8) and (2.9) we obtain the following theorem. 
Theorem 2.4. Let $\lambda \in(0,1)$. Then, for all $z \in \mathbb{C}$, with $z \neq 0,-1,-2, \ldots, \frac{1}{\lambda}, \frac{1}{\lambda}+1, \frac{1}{\lambda}+2, \ldots$,

$$
\begin{aligned}
\Gamma_{\lambda}(z) & =\frac{\lambda^{-z}}{z\left(\frac{1}{\lambda}-z\right)} \frac{1}{\Gamma\left(\frac{1}{\lambda}\right)} e^{-\frac{\gamma}{\lambda}} \prod_{n=1}^{\infty}\left\{e^{\frac{1}{n \lambda}}\left(1+\frac{z}{n}\right)^{-1}\left(1+\frac{\frac{1}{\lambda}-z}{n}\right)^{-1}\right\} \\
& =\frac{\lambda^{-z}}{z\left(\frac{1}{\lambda}-z\right)} \frac{1}{\Gamma\left(\frac{1}{\lambda}\right)} \prod_{n=1}^{\infty}\left\{\left(1+\frac{1}{n}\right)^{\frac{1}{\lambda}}\left(1+\frac{z}{n}\right)^{-1}\left(1+\frac{\frac{1}{\lambda}-z}{n}\right)^{-1}\right\} .
\end{aligned}
$$

It follows from Theorem 2.4 that

$$
\begin{aligned}
\Gamma_{\lambda}(z) & =\frac{\lambda^{-z}}{\Gamma\left(\frac{1}{\lambda}\right)} \frac{1}{z\left(\frac{1}{\lambda}-z\right)} \prod_{n=1}^{\infty}\left\{\left(\frac{n+1}{n}\right)^{\frac{1}{\lambda}}\left(\frac{n}{n+z}\right)\left(\frac{n}{n+\frac{1}{\lambda}-z}\right)\right\} \\
& =\frac{\lambda^{-z}}{\Gamma\left(\frac{1}{\lambda}\right)} \frac{1}{z\left(\frac{1}{\lambda}-z\right)} \lim _{m \rightarrow \infty}\left\{m^{\frac{1}{\lambda}} \frac{(m-1) !}{(1+z) \cdots(m-1+z)}\right. \\
& \left.\times \frac{(m-1) !}{\left(1+\frac{1}{\lambda}-z\right) \cdots\left(m-1+\frac{1}{\lambda}-z\right)}\right\} \quad m^{\frac{1}{\lambda}}((m-1) !)^{2} \\
& =\frac{\lambda^{-z}}{\Gamma\left(\frac{1}{\lambda}\right)} \lim _{m \rightarrow \infty} \frac{1+z(1+z) \cdots(m-1+z)\left(\frac{1}{\lambda}-z\right)\left(1+\frac{1}{\lambda}-z\right) \cdots\left(m-1+\frac{1}{\lambda}-z\right)}{z(1+z)} .
\end{aligned}
$$

Therefore, by (2.10), we obtain the following theorem.

Theorem 2.5. Let $\lambda \in(0,1)$. Then, for all $z \in \mathbb{C}$, with $z \neq 0,-1,-2, \ldots, \frac{1}{\lambda}, \frac{1}{\lambda}+1, \frac{1}{\lambda}+2, \ldots$,

$$
\Gamma_{\lambda}(z)=\frac{\lambda^{-z}}{\Gamma\left(\frac{1}{\lambda}\right)} \lim _{n \rightarrow \infty} \frac{n^{\frac{1}{\lambda}}((n-1) !)^{2}}{z(1+z) \cdots(n-1+z)\left(\frac{1}{\lambda}-z\right)\left(1+\frac{1}{\lambda}-z\right) \cdots\left(n-1+\frac{1}{\lambda}-z\right)} .
$$

Let $\lambda \rightarrow 1-$ in $(2.11)$. Then

$$
\begin{aligned}
\Gamma_{1}(z) & =\lim _{n \rightarrow \infty} \frac{n((n-1) !)^{2}}{z(1+z) \cdots(n-1+z)(1-z)(2-z) \cdots(n-1-z)(n-z)} \\
& =\lim _{n \rightarrow \infty} \frac{((n-1) !)^{2}}{z\left(1^{2}-z^{2}\right)\left(2^{2}-z^{2}\right) \cdots\left((n-1)^{2}-z^{2}\right)} \\
& =\frac{1}{z} \lim _{n \rightarrow \infty} \frac{1}{\left(1-\left(\frac{z}{1}\right)^{2}\right)\left(1-\left(\frac{z}{2}\right)^{2}\right) \cdots\left(1-\left(\frac{z}{n-1}\right)^{2}\right)} \\
& =\frac{1}{z} \prod_{n=1}^{\infty}\left(1-\frac{z^{2}}{n^{2}}\right)^{-1} .
\end{aligned}
$$

By Lemma 2.1, passing to the limit as $\lambda \rightarrow 1-$, we obtain

$$
\Gamma_{1}(z)=\frac{\Gamma(z) \Gamma(1-z)}{\Gamma(1)}=\frac{\pi}{\sin \pi z} .
$$

Therefore, by (2.12) and (2.13), we obtain the following corollary.

Corollary 1. For all $z \in \mathbb{C} \backslash \mathbb{Z}$,

$$
\frac{\pi z}{\sin \pi z}=\prod_{n=1}^{\infty}\left(1-\frac{z^{2}}{n^{2}}\right)^{-1}
$$

In connection with equation (1.4), it is natural to define the degenerate beta functions by

$$
B_{\lambda}(\alpha, \beta)=\frac{\Gamma_{\lambda}(\alpha) \Gamma_{\lambda}(\beta)}{\Gamma_{\lambda}(\alpha+\beta)}
$$

where $\alpha, \beta \neq 0,-1,-2, \ldots \frac{1}{\lambda}, \frac{1}{\lambda}+1, \frac{1}{\lambda}+2, \ldots$

Using Lemma 2.1, Theorem 2.3, and the first identity in Theorem 2.4, we immediately derive the following expressions for the degenerate beta function. 
Theorem 2.6. Let $\lambda \in(0,1)$. Then, for any $\alpha, \beta \neq 0,-1,-2, \ldots \frac{1}{\lambda}, \frac{1}{\lambda}+1, \frac{1}{\lambda}+2, \ldots$, the following equalities hold:

$$
\begin{aligned}
& B_{\lambda}(\alpha, \beta)=\frac{1}{\Gamma\left(\frac{1}{\lambda}\right)} B(\alpha, \beta) \frac{\Gamma\left(\frac{1}{\lambda}-\alpha\right) \Gamma\left(\frac{1}{\lambda}-\beta\right)}{\Gamma\left(\frac{1}{\lambda}-\alpha-\beta\right)}=\frac{e^{-\frac{\gamma}{\lambda}}(\alpha+\beta)\left(\frac{1}{\lambda}-\alpha-\beta\right)}{\Gamma\left(\frac{1}{\lambda}\right) \alpha \beta\left(\frac{1}{\lambda}-\alpha\right)\left(\frac{1}{\lambda}-\beta\right)}
\end{aligned}
$$

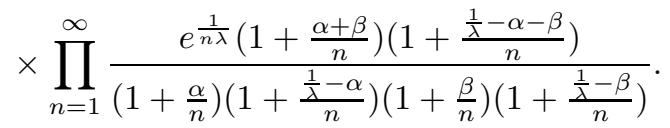

In particular, if $\alpha=m$ and $\beta=n$ are positive integers, then we have the following expression.

$$
B_{\lambda}(m, n)=\frac{(1)_{m+n+1, \lambda}}{(1)_{m+1, \lambda}(1)_{n+1, \lambda}} B(m, n) .
$$

\section{FURTHER REMARKS}

As is traditional, let $\int_{\infty}^{(0+)}$ (respectively, $\int_{-\infty}^{(0+)}$ ) denote the integration along the path that starts at infinity (respectively, at the negative infinity), encircles the origin in the counter-clockwise direction and returns to the starting point. Assume that $0<\lambda<1,0<\operatorname{Re}(s)<\frac{1}{\lambda}$.

As we noted in (2.1), we have

$$
\lambda^{s} \Gamma_{\lambda}(s)=\int_{0}^{\infty} t^{s-1}(1+t)^{-\frac{1}{\lambda}} d t
$$

We now consider the integral given by

$$
\int_{C} \frac{z^{s-1}}{(1-z)^{\frac{1}{\lambda}}} d z
$$

Here $C$ is the path that moves along the negative real axis from $-R$ to $-\delta$, along the circle of radius $\delta(<1)$ in the counter-clockwise direction, and then comes along the negative real axis from $-\delta$ to $-R$. Then the integral in (3.2) is equal to

$$
\int_{R}^{\delta} \frac{t^{s-1} e^{-i \pi s}}{(1+t)^{\frac{1}{\lambda}}} d t+\int_{-\pi}^{\pi} \frac{i R^{s} e^{i s \theta}}{\left(1-R e^{i \theta}\right)^{\frac{1}{\lambda}}} d \theta+\int_{\delta}^{R} \frac{t^{s-1} e^{i \pi s}}{(1+t)^{\frac{1}{\lambda}}} d t .
$$

Let $R \rightarrow \infty$ and $\delta \rightarrow 0$. Then the second integral in (3.3) tends to zero, and hence we obtain

$$
\lambda^{s} \Gamma_{\lambda}(s)=\int_{0}^{\infty} t^{s-1}(1+t)^{-\frac{1}{\lambda}} d t=\frac{1}{2 i \sin \pi s} \int_{-\infty}^{+0} \frac{z^{s-1}}{(1-z)^{\frac{1}{\lambda}}} d z .
$$

We note here that the rightmost integral in (3.4) vanishes at $s=1,2,3, \ldots$ and it does not vanish at $s=0,-1,-2,-3, \ldots$ Thus, this gives an analytic continuation $\Gamma_{\lambda}(s)$ to a meromorphic function on $\mathbb{C}$ with simple poles at $s=0,-1,-2,-3, \ldots$ Further, in view of the symmetry of $\lambda^{-s} \Gamma_{\lambda}(s)$ under the transformation $s \rightarrow \frac{1}{\lambda}-s$, this function has also simple poles at $\frac{1}{\lambda}, \frac{1}{\lambda}+1, \frac{1}{\lambda}+2, \ldots$ As we can see from Theorem 2.4, s=0,-1,-2,-3, .., $\frac{1}{\lambda}, \frac{1}{\lambda}+1, \frac{1}{\lambda}+2, \ldots$ are all the poles of $\Gamma_{\lambda}(s)$.

Finally, by replacing $z$ by $-z$ in (3.4), we also have the expression

$$
\lambda^{s} \Gamma_{\lambda}(s)=\frac{i}{2 \sin \pi s} \int_{\infty}^{+0} \frac{(-z)^{s-1}}{(1+z)^{\frac{1}{\lambda}}} d z .
$$

\section{CONCLUSION}

The degenerate Bernoulli and Euler polynomials were introduced by Carlitz as degenerate versions of ordinary Bernoulli and Euler polynomials, respectively. In recent years, the study of degenerate versions of some special polynomials and numbers regained interests of many mathematicians and tuned out to be very useful and fruitful. The idea of investigating diverse degenerate versions is not just limited to polynomials but can be extended to transcendental functions. Indeed, the degenerate gamma functions were introduced in [6] as a degenerate version of the usual gamma function (see also [7]). 
In this paper, we derived several basic properties of the degenerate gamma functions. Firstly, we noted that, by making use of (2.1), the degenerate gamma function $\Gamma_{\lambda}(s)$ initially defined for $0<\operatorname{Re}(s)<\frac{1}{\lambda}$ can be analytically continued to a meromorphic function on $\mathbb{C}$, except for simple poles at

$$
s=0,-1,-2, \ldots \frac{1}{\lambda}, \frac{1}{\lambda}+1, \frac{1}{\lambda}+2, \ldots
$$

Secondly, we derived the difference formula in Theorem 2.2, determined their values at positive integers in Theorem 2.3, and found several expressions by making use of the Weierstrass and Euler formulas for the usual gamma function in Theorems 2.4 and 2.5. Thirdly, we defined the degenerate beta functions and obtained some expressions for them. Lastly, we obtained an integral representation for the degenerate gamma functions as an integral along a Hankel contour.

\section{REFERENCES}

[1] C. Adiga, "A Note on the $q$-Gamma and $q$-Beta Functions," Math. Balkanica (N.S.) 9 (1), 35-41 (1995).

[2] T. H. Gronwall, "The Gamma Function in the Integral Calculus," Ann. of Math. (2) 20, 35-124 (1918).

[3] G. D. Birkhoff, "Note on the Gamma Function," Bull. Amer. Math. Soc. 20 (1), 1-10 (1913).

[4] J. L. W. V. Jensen and T. H. Gronwall, "An Elementary Exposition of the Theory of the Gamma Function," Ann. of Math. (2) 17 (3), 124-166 (1916).

[5] W. A. Khan, "A New Class of Degenerate Frobenius-Euler-Hermite Polynomials," Adv. Stud. Contemp. Math. (Kyungshang) 28 (4), 567-576 (2018).

[6] T. Kim and D. S. Kim, "Degenerate Laplace Transform and Degenerate Gamma Function," Russ. J. Math. Phys. 24 (2), 241-248 (2017).

[7] T. Kim and G.-W. Jang, "A Note on Degenerate Gamma Function and Degenerate Stirling Number of the Second Kind," Adv. Stud. Contemp. Math. (Kyungshang) 28 (2), 207-214 (2018).

[8] D. S. Kim, T. Kim, and G.-W. Jang, "A Note on Degenerate Stirling Numbers of the First Kind," Proc. Jangjeon Math. Soc. 21 (3), 393-404 (2018).

[9] T. Kim, D. S. Kim, and H.-I. Kwon, "A Note on Degenerate Stirling Numbers and Their Applications," Proc. Jangjeon Math. Soc. 21 (2), 195-203 (2018).

[10] T. Kim, " $\lambda$-Analogue of Stirling Numbers of the First Kind," Adv. Stud. Contemp. Math. (Kyungshang) 27 (3), 423-429 (2017).

[11] T. Kim, D. S. Kim, J. Kwon, and H. Lee, "A Note on Degenerate Gamma Random Variables," Revista Edu. 388 (4), 39-44 (2020).

[12] Y. Kim, B. M. Kim, L.-C. Jang, and J. Kwon, "A Note on Modified Degenerate Gamma and Laplace Transformation," Symmetry (10), 471 (2018).

[13] D. V. Kruchinin and V. V. Kruchinin, "Explicit Formula for Reciprocal Generating Function and Its Application," Adv. Stud. Contemp. Math. (Kyungshang) 29 (3), 365-372 (2019).

[14] E. L. Post, "The Generalized Gamma Functions," Ann. of Math. (2) 20 (3), 202-217 (1919).

[15] G. Rahman, K.S. Nisar, T. Kim, S. Mubeen, and M. Arshad, "Inequalities Involving Extended $k$-Gamma and k-Beta Functions," Proc. Jangjeon Math. Soc. 21 (1), 143-153 (2018).

[16] E. D. Rainville, Special Functions (The Macmillan Co., New York, 1960).

[17] E. D. Rainville, Special Functions (Reprint of 1960 first edition. Chelsea Publishing Co., Bronx, N.Y., 1971).

[18] E. D. Rainville, "Recent Publications and Presentations: Solved Problems: Gamma and Beta Functions, Legendre Polynomials, Bessel Functions," Amer. Math. Monthly 71 (9), 1063 (1964).

[19] G. Rasch, "Notes on the Gamma-Function," Ann. of Math. (2) 32 (3), 591-599 (1931).

[20] L. M. Upadhyaya, "On the Degenerate Laplace Transforms IV," Inter. J. Eng. Sci. Res. 6 (2), 198-209 (2018).

[21] P. Vassilev, "On an Estimate from above for the Remainder Sum of Certain Series Related to Euler's Gamma Function," Adv. Stud. Contemp. Math. (Kyungshang) 24 (1), 109-128 (2014).

[22] E. T. Whittaker and G. N. Watson, A Course of Modern Analysis. An Introduction to the General Theory of Infinite Processes and of Analytic Functions; with an Account of the Principal Transcendental Functions (Reprint of the fourth (1927) edition. Cambridge Mathematical Library. Cambridge University Press, Cambridge, 1996). 